\title{
Lignin as A Natural Sunscreen-An Overview
}

\author{
Hasan Sadeghifar* \\ Hasan Sadeghifar, Hollingworth \& Vose, USA \\ *Corresponding author: Hasan Sadeghifar, Hollingworth \& Vose, 219 Townsend road, Graton, MA 01450, USA
}

\section{ARTICLE INFO}

Received: 慧 March 28, 2020

Published: April 09, 2020

Citation: Hasan Sadeghifar. Lignin as A Natural Sunscreen-An Overview. Biomed J Sci \& Tech Res 27(1)-2020. BJSTR. MS.ID.004430.

\begin{abstract}
Lignin is the main natural phenolic bio-polymer available in the market as a byproduct of pulp and paper industries. Having UV chromophore functional groups makes it an ideal light blocker in the ultraviolet (UV) spectrum. Both in pure form and as a mixture with commercial sunscreen, lignin indicated high potential as a bio-based sunscreen. Due to its complex structure, impurities, and brownish color, more work is needed to make lignin an ideal bio-based sunscreen.
\end{abstract}

Keywords: UV: Ultraviolet; LS: Lignosulfonate; SPF: Sun Protection Factor

\section{Introduction}

Despite the fact that sun light is necessary to generate vitamin D for the body, prolonged sun light UV exposure can cause significant damage to eyes and skin [1]. Based on the wave length, UV radiation is classified into three main categories; UV-A, UV-B and UV-C. UV-B (290-320 nm) and UV-A (320-400 nm) are able to pass through earth's ozone layer and have significant effects on the epidermis layer of the skin $[2,3]$. The UV radiation generates free radicals which can lead to skin aging, skin damage, and in some cases, skin cancer [4]. The main method for preventing the free radicals' activity and skin damage is by blocking UV radiation. Sun blocker creams in the market use minerals such as zinc oxide $\left(\mathrm{ZnO}_{2}\right)$ and titanium dioxide $\left(\mathrm{TiO}_{2}\right)$ along with a wide range of organic chemicals to block UV radiation. Due to the negative side effects of synthetic sun blockers on skin tissue, the use of natural sun blockers has been garnering attention. Many natural polyphenol extracts have been tested as UV blockers. However, most of them are small molecules with low purity, have poor photo-stability [4], and cannot block the full UV spectrum [5]. Therefore, more stable natural macromolecular sunscreens are needed. Lignin is the most natural aromatic polymer on earth and is readily available as a by-product of pulp and papermaking industries. Depending on the pulping process, two categories of lignin are available: alkali lignin and sulfonated lignin. Alkali lignin is not soluble in water but lignosulfonate dissolves easily in water due to the presence of hydrophilic functional groups. Chemical structures of the two extracted lignin types are illustrated in Figure 1. Lignin has a complex three-dimensional structure containing high carbon content, UV chromophore groups, and aromatic rings of hydroxyl and methoxyl groups along with double bonds and carbonyl functional groups. These properties make it a good candidate as a UV blocker agent and antioxidant [69]. UV protection is generally calculated as a Sun Protection Factor (SPF) which is defined by the following equation:

$$
S P F=\sum_{290}^{400} E_{\lambda} S_{\lambda} / \sum_{290}^{400} E_{\lambda} S_{\lambda} T_{\lambda}
$$

$E_{\lambda}=$ CIE erythemal spectral effectiveness, $S_{\lambda}=$ solar spectral irradiance, and $\mathrm{T}_{\lambda}=$ spectral transmittance of the sample [9]. The percentage of UV protection is equal to $100-(100 / S P F)$. For example, a material with SPF 15 means it can protect $100-(100 / 15)=$ $93.33 \%$ of UV-B light. Addition of technical lignin will significantly increase SPF of sun lotions and broad-spectrum UV-absorbtion [10] as well as enhance antioxidant properties [10]. Similar to traditional sunscreen, the application of lignin as a UV blocker is in the form of a mixture with a base lotion or cream. However, pure cream containing a low percentage of lignin has indicated low SPF [9]. In contrast, addition of too much hydrophilic lignin to cream will cause demulsification, which is a challenge for this application. Furthermore, technical lignin derived from the pulping process is a dark heterogeneous material containing minerals and organic impurities. To address these challenges, lignin modification, purification, fractionation, and extraction methods are proposed to improve UV blocking performance and practical application. 


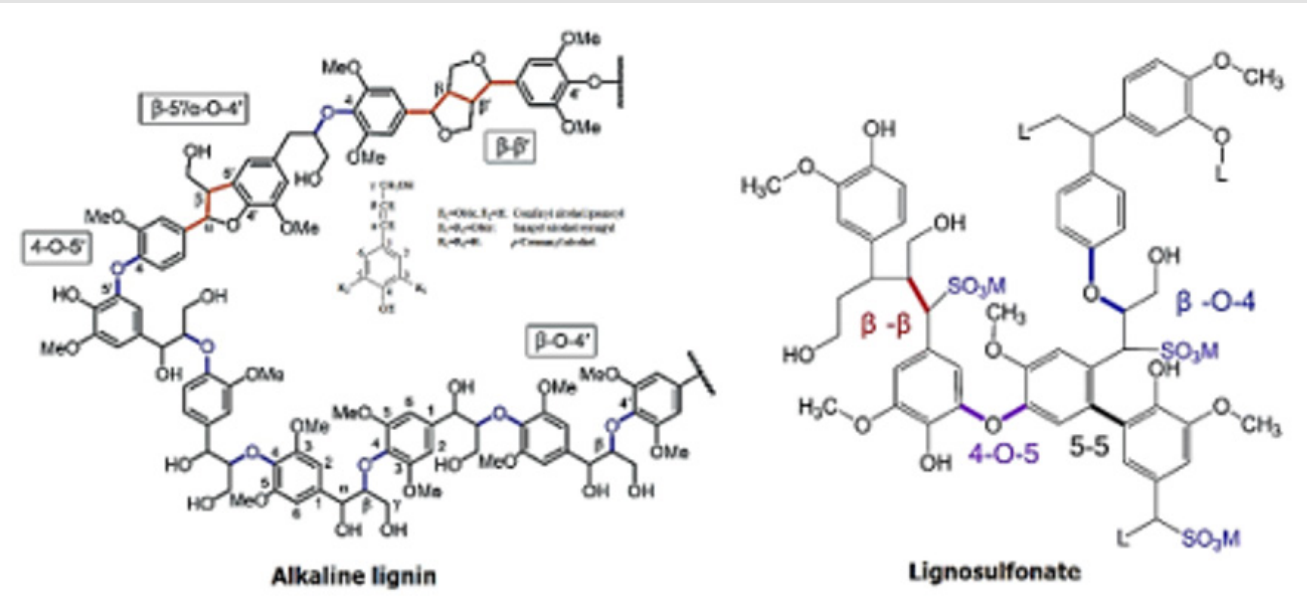

Figure 1: Model structure for alkali extracted and sulfonated lignin and their phenyl propane precursor structure.

\section{Lignin as Natural Sunscreen Cream}

Evaluation of alkaline lignin after wash and purification for development of high-performance broad-spectrum sunscreen is reported [11]. Lignin was added into several commercial sunscreen products. Significant enhancements in ultraviolet (UV) absorbance was reported. Adding $2 \mathrm{wt} \%$ lignin to SPF 15 sunscreen doubled its SPF to 30. Adding 10wt\% lignin increased its SPF to 50. Enhanced sunscreen performance after radiation with UV light was also reported. After 2 hours of UV radiation, UV absorbance of $10 \mathrm{wt} \%$ lignin SPF 15 sunscreen increased dramatically. This has been attributed to certain synergistic effects between lignin and other ingredients in the sunscreen lotions, as well as the antioxidant property of lignin. Lignin-based sunscreen creams were prepared by blending $10 \%$ lignin (or modified lignin colloidal particle) with $90 \%$ pure NIVEA cream at $300 \mathrm{rpm}$ for 20 hours at room temperature in darkness [9]. The critical wavelength $(\lambda c)$ of the optimal sample was $\sim 375 \mathrm{~nm}$, while the UVA/UVB ratio reached 0.84. By using $130 \mathrm{~nm}$ of colloidal lignin particle, SPF of the cream was increased to 56 . Superior photo-stability was also reported due to the three-dimensional network structure of lignin.

In an effort to investigate their UV blocking performance, Lignin samples were blended with a commercial sunscreen (BIOTHERM SPF 15) [12]. When 3 wt.\% lignin was added, SPF of the sunscreen approximately doubled. Adding lignin into a sunscreen indicated better results than blending lignin in a pure hand cream due to the synergistic effects that were reported [11]. Additionally, when lignin-containing sunscreen lotions were exposed to UV radiation for 2 hours, their sunscreen performance drastically improved. The sun lotions without lignin, however, exhibited a marginal change in UV absorbance. Addition of $1 \mathrm{wt} \%$ lignin and 40 minutes of UV radiation exposure increased the SPF value of the sunscreen from 8.4 to approximately 40 .

In another effort to use lignin micrometer particles from organic acid lignin [13], there was indication of significant increase to the
SPF values of pure lotion by $2.80-3.53$ at $5 \%$ dosage. Furthermore, the UVA/UVB ratio is reported to be in the range of $0.69-0.72$ for this type of lignin, indicating that they exhibit superior properties. It was claimed that lignin particles, with excellent antioxidant and UV protection capacities, are a natural source for sunblock cosmetics. In another study, Lignosulfonate (LS) was applied to modify $\mathrm{TiO}_{2}$ as a mineral sunscreen [14]. The results showed that esterification occurred between the carboxyl groups of lignin and the hydroxyl groups on the surface of $\mathrm{TiO}_{2}$. The coated lignin on the $\mathrm{TiO}_{2}$ surface not only improved the dispensability of $\mathrm{TiO}_{2}$ in the substrates, but also significantly boosted its UV-blocking ability. $\mathrm{TiO}_{2}$ coated with lignin nanocomposites was applied in a pure cream and the sunscreen performance was studied, with pure $\mathrm{TiO}_{2}$ as control. The sun protection factor (SPF) values of creams containing 5, 10 , and $20 \mathrm{wt} \%$ lignin on $\mathrm{TiO}_{2}$ were 16,26 , and 48, respectively. The same study is reported using lignin and zinc oxide $(\mathrm{ZnO})$ as another mineral sunscreen [15]. This coating broadened UV protection to cover the full spectrum of UVB and UVC. The SPF values improved in a range between 4 and 13. Lastly, applying lignin nanoparticle from rice husk into moisturizing cream (NIVEA) at concentrations of 1 and $5 \mathrm{wt} \%$ is reported to make a lignin-based sunscreen $[16,17]$. At 5 wt $\%$ lignin nanoparticles, the cream exhibited SPF values about twice as high as those of the pure cream. When lignin nanoparticles were added into commercial sunscreen (5 wt \%), UV protection factor increased by a factor of five overall (from SPF 5.4 to SPF 30.0).

\section{Conclusion}

Lignin as a bio-based phenolic material indicates high potential as a natural wide spectrum (UVA, UVB) sunscreen. Mixing lignin with lotion and cream results in high sun protection factor (SPF). Especially when mixed with other commercial sunscreens in the market, and in synergistic effect with other ingredients, a low dosage of lignin dramatically increases the SPF and strengthens the sunscreen's properties. 


\section{Competing Interests}

The authors declare that they have no competing interests.

\section{Acknowledgement}

Not applicable.

\section{References}

1. Hanson KM, Gratton E, Bardeen CJ (2006) Sunscreen enhancement of UV-induced reactive oxygen species in the skin. Free Radical Biol Med 41: $1205-1212$.

2. Rass K, Reichrath J (2008) UV damage and DNA repair in malignant melanoma and non-melanoma skin cancer. Adv. Exp. Med Biol 624: 162178.

3. Deng Y, Ediriwickrema A, Yang F, Lewis J, Girardi, M, et al. (2015) A sun block based on bio-adhesive nanoparticles. Nat Mater 14: 1278-1285.

4. Nichols JA, Katiyar SK (2010) Skin photoprotection by natural polyphenols anti-inflammatory: antioxidant and DNA repair mechanisms. Arch Dermatol Res 302: 71-83.

5. Sadeghifar H, Venditti R, Jur J, Gorga RE, Pawlak JJ (2017) CelluloseLignin Biodegradable and Flexible UV Protection Film, ACS Sustainable Chem Eng 5: 625-631.

6. Lou HM, Lai HR, Wang MX, Pang YX, Yang DJ, et al. (2013) Preparation of lignin-based superplasticizer by graft sulfonation and investigation of the dispersive performance and mechanism in a cementitious system. Ind Eng Chem Res 52: 16101-16109.

7. Argyropoulos DS (1994) Quantitative phosphorus-31 NMR analysis of lignin's: a new tool for the lignin chemist. J Wood Chem Technol 14: 4563.

ISSN: 2574-1241

DOI: $10.26717 /$ BJSTR.2020.27.004430

Hasan Sadeghifar. Biomed J Sci \& Tech Res

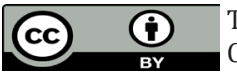

This work is licensed under Creative Commons Attribution 4.0 License

Submission Link: https://biomedres.us/submit-manuscript.php
8. Barsberg S, Elder T, Felby C (2003) Lignin-Quinone Interactions: Implications for Optical Properties of Lignin. Chem Mater 15: 649-655.

9. Dorrestijn E, Laarhoven L, Arends I, Mulder P (2000) The occurance and reactivity of phenoxyl linkages in lignin and low rank coal. J Anal Appl Pyrolysis 54:153-192

10. Qian Y, Qiu X, Zhu S (2015) Lignin: a nature-inspired sun blocker for broad spectrum Sunscreens. Green Chem. 17: 320-324.

11. Sadeghifar H, Argyropoulos DS (2015) Correlations of the antioxidant properties of softwood kraft lignin fractions with the thermal stability of its blends with polyethylene, ACS Sustainable Chemistry \& Engineering 3: 349-356.

12. Qian Y, Qiu XS, Zhu S (2016) Sunscreen performance of lignin from different technical resources and their general synergistic effect with synthetic sunscreens, ACS Sustain. Chem Eng 4: 4029-4035.

13. Wu Y, Qian Y, Lou H, Yang D, Qiu X (2019) Enhancing the Broad-Spectrum Adsorption of Lignin through Methoxyl Activation, Grafting Modification, and Reverse Self- Assembly. ACS Sustainable Chem Eng 7: 15966-15973.

14. Lee SC, Tran TMT, Choi JW, Wona K (2019) Lignin for white natural sunscreens, International Journal of Biological Macromolecules 122: 549-554.

15. Li SX, Li MF, Bain J, Wu XF, Peng F, et al. (2019) Preparation of organic acid lignin submicrometer particle as a natural broad-spectrum photoprotection agent. International Journal of Biological Macromolecules 132: 836-843.

16. Yu J, Li L, Qian Y, Lou H, Yang D, et al. (2018) Dioxide Nanocomposites for Developing Natural Sunscreens. Ind Eng Chem Res 57: 15740-15748.

17. Lee SC, Yoo E, Lee SH, Won K (2010) Preparation and Application of Light-Colored Lignin Nanoparticles for Broad-Spectrum Sunscreens. Polymers 12: 699-713.

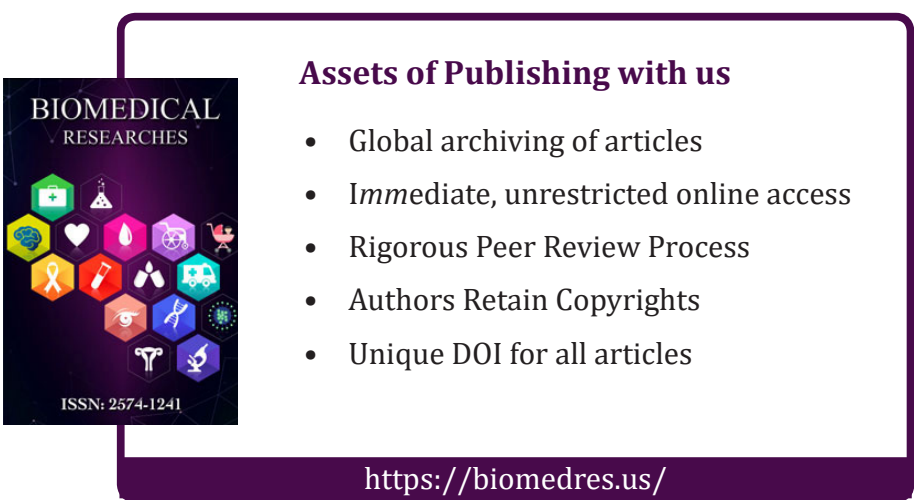

